\title{
Diesseits und jenseits der Oder: Judith Hermanns literarische Auseinandersetzung mit Theodor Fontane in Sommerhaus, spa"ter
}

Claudia Gremler

\begin{abstract}
Judith Hermann's works have attracted considerable criticism for their supposedly slight portrayal of passively drifting characters and for their alleged failure to engage with the socio-political realities of contemporary life in the Berlin Republic. Only very recently have scholars paid attention to the hidden concern with memory expressed in her books, and have set out to examine their intertextual depth. This paper explores these previously neglected historical references in Summerhouse, later and analyses the book's intricate intertextual allusions with specific reference to Theodor Fontane's works. It examines how the tentative existence, which Hermann's characters experience, is the product of a hesitant and fruitless confrontation with questions of German history and nationhood. Using pervasive water imagery, Hermann shows present-day Germany as a continually contested territory with a fluid identity shaped by an abundance of conflicting narratives. In this context, the allusions to Fontane as a representative of the Wilhelminian period serve as references to a continuing German tradition of repression and marginalisation. At the same time, Hermann recognises Fontane's ambivalent political stance combining elements of social criticism with a general endorsement of social order. Ultimately, the seemingly indifferent attitude of Hermann's characters and the elegiac style used to portray them, emerge as a distancing mechanism that functions as a postmodern variant of Fontane's irony and is shaped by a similar sense of skepticism towards developments in German society and national history.
\end{abstract}

Keywords Judith Hermann . Sommerhaus, spa“ter . Theodor Fontane . Effi Briest . Contemporary German literature . National identity . Memory studies . Gender studies . Intertextuality

C. Gremler (\&) Aston University, Birmingham, UK e-mail: C.Gremler@aston.ac.uk

\section{3}

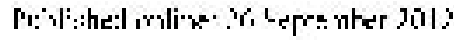


Die Autorin Judith Hermann, deren literarisches Debu“t, die Erza“hlsammlung Sommerhaus, spa"ter (1998) von Publikum und Rezensenten gleichermaßen begeistert aufgenommen worden war, musste nach Erscheinen ihres zweiten und dritten Buches zunehmend mit ablehnenden Reaktionen sowohl aus den Feuilletons als auch vonseiten der Literaturwissenschaft ka“mpfen. In pra“skriptiven Urteilen, die sich ru“ckwirkend auch auf ihren Erstling beriefen, wurde ihr vorgeworfen, sie habe sich einer politische und historische Aspekte vernachla“ssigenden Gegenwartsschilderung von popkultureller Simplizita“t verschrieben und berichte aus narzisstischer Perspektive desinteressiert u“ber ,die Ziellosigkeit als Lebensstil und die Leere als Lebensinhalt“ ( (Lo“ffler 2009).

Hermanns vermeintlich geschichtslose und dekontextualisierte Darstellung galt Kritikern als besonders verwerflich, weil diese Autorin, aus deren Erza“hlungen Kritikern der „Sound einer neuen Generation“ (Literarisches Quartett 2006, Bd. III, 151) entgegento“nte, erfolgreich als Repra“sentantin ihrer Altersgenossen vermarktet worden war und ihre Werke daher als stellvertretend für einen Gesamttrend angesehen wurden, der sich auffallend von der auf Erinnerungsvorga“nge fokussierten Tradition der deutschen Nachkriegsliteratur abzuheben schien.

Unter Feuilletonisten und Literaturwissenschaftlern herrschte eine auffallend emotionsbeladene Unzufriedenheit daru“ber, dass Hermanns Werke sprachlich zwar einen faszinierenden „elegisch[en] Grundton“ (Schulze 1998) aufweisen, der durch seine „klare Sprache einen tiefen Sog [entfaltet]“ (Illies 1998), dass aber inhaltlich in ihren Büchern orientierungslose Figuren wahllos durch die Welt ziehen oder ein nahezu konturenloses Berlin bewohnen. Diese Charaktere schienen passiv immer auf den na“chsten folgenlosen thrill zu warten, und Hermanns Zugeho“rigkeit zu einer unkritischen „Erlebnisgesellschaft“ “ ${ }^{2}$ wurde deshalb bald zum Konsens erkla“rt.

Diese Einscha“tzung von Hermanns Werken verstellte lange Zeit den Blick auf diejenigen Dimensionen in ihrem Schreiben, die sich durchaus mit Fragen nach der Geschichte und nach sozialen Kontexten befassen und sich zugleich auch mit der literarischen Tradition auseinandersetzen. Inzwischen setzt sich erfreulicherweise in der wachsenden Hermann-Forschung eine neue Herangehensweise an ihre Erza“hlungen durch, die den zweifellos vorhandenen ,,concern with memory“ (Taberner 2005,20) und die eng damit verbundene ,intertextual depth“ (Shafi 2009, 345) ihrer Werke sta“rker in den Vordergrund ru“ckt.

Diese beiden Aspekte bedingen sich zu einem gewissen Grad gegenseitig, denn zur sozio-historischen Kontextualisierung ihrer Texte setzt Hermann ein dichtes Netz intertextueller Bezu"ge zum literarischen Kanon ein, die dazu dienen, eine als problematisch begriffene deutsche Traditionslinie vom Kaiserreich zur Berliner Republik nachzuzeichnen. Eine zentrale Rolle nimmt dabei die Auseinandersetzung mit Theodor Fontanes Werken ein.

1

Vgl. etwa die am 31.01.2003 von Elmar Krekeler in der Welt gestellte ungeduldige Frage, ,,warum man diesen Figuren eigentlich sechzig Seiten über die Schulter sehen soll, statt sie zu schu"tteln und aufzuwecken".

²So vor allem Biendarra (2004) unter Berufung auf Gerhard Schulze. 
Besonders deutlich zeigt sich Hermanns Bezugnahme auf diesen einflussreichen Vertreter der deutschen Literatur im neunzehnten Jahrhundert in der Erza“hlung „Rote Korallen“. Dieser Text ero“ffnet den Band und scheint zuna“chst in mehrfacher Hinsicht eine Sonderstellung in Hermanns Werk einzunehmen. Anders als bei den mehrheitlich im Berlin der Gegenwart verorteten u“brigen Erza“hlungen in Sommerhaus, spa“ter ist fu“r „,Rote Korallen“ “ das Sankt Petersburg der vorigen Jahrhundertwende als Schauplatz gewa"hlt worden. Die Erza“hlung geho“rt zu den am meisten beachteten des Bandes und ist sogar für den Einsatz im Schulunterricht empfohlen worden (Burtscher 2002). Dem Hinweis der Autorin, diese Erza“hlung als wegweisendes „Motto“ fu“r das gesamte Buch zu verstehen (Geiger 2004, 49), ist man jedoch kaum gefolgt. Deshalb ist ein prima“r von diesem Text ausgehender Zugang zum Buch, der diese Erza“hlung nicht als Ausnahme, sondern als paradigmatisch für Hermanns Werk betrachtet und unweigerlich den Schwerpunkt der Interpretation auf historische und intertextuelle Dimensionen legen muss, bedauerlicherweise bisher ausgeblieben. Diese Forschungslu“cke beabsichtigt der vorliegende Beitrag zu schließen.

In „,Rote Korallen“ w werden mehrere Zeitebenen entworfen, die durch die Figur der Ich-Erza“hlerin und Protagonistin verbunden sind. Im Zentrum der von ihr erza“hlten

Binnenhandlung steht das Schicksal ihrer rebellischen Urgroßmutter im vorrevolutiona“ren Russland. Von ihrem wenig einfu“hlsamen Ehemann allein gelassen und von Heimweh geplagt, gibt die scho"ne junge Frau dem Werben verschiedener Liebhaber nach, von denen einer ihr ein auffa“lliges rotes Korallenarmband schenkt. Nach der Ru“ckkehr des Gatten von seinen Reisen offenbart sich deutlich die innere Distanz zwischen den Eheleuten. Daraufhin zeigt die Urgroßmutter provokativ als demonstratives Zeichen ihrer Untreue das Korallenarmband vor, das „rot wie die Wut“ (SS, 15) leuchtet. Der gedemu“tigte Ehemann stirbt im Duell mit dem Liebhaber. Daraufhin verla“sst die junge Witwe Sankt Petersburg und kehrt nach Berlin zuru“ck.

Diese Geschichte der ehebrecherischen Urgroßmutter, die mit ihrer bewussten Auflehnung gegen die Regeln der bürgerlichen Ehe auffallend subversiv und modern erscheint, weist deutliche intertextuelle Bezu"ge auf zu dem kanonischen Ehebruchstext der deutschen Literatur im neunzehnten Jahrhundert. Die bürgerlichpatriarchalen ma“nnlichen Figuren verhalten sich bei Hermann nicht zufa“llig so, „wie wir es aus Fontanes Romanen gewohnt sind“ " (Stephan 2002, 550), vielmehr pra“sentiert sich die Geschichte der Urgroßmutter als ein klarer Gegenentwurf zu Fontanes Romanwelt im Allgemeinen und zu Effi Briest im Besonderen.

Schon bei der Erstlektu“re von „Rote Korallen“ fallen deutliche Parallelen auf, die auch ohne explizite intertextuelle Markierung ${ }^{4}$ eine bewusste Bezugnahme auf Effi Briest signalisieren. Wie in Fontanes Ehebruchsgeschichte verla“sst auch in Hermanns Erza“hlung eine junge Ehefrau ihre Heimat im preußischen Kernland und geht mit ihrem Gatten in den ihr unbekannten Osten-und Effi bereitet sich auf den

${ }^{3}$ SS wird als Sigel für Sommerhaus, spa“ter verwendet. ${ }^{4}$ Zur Frage der Markierung von Intertextualita"t vgl. Broich (1985). 
Umzug nach Pommern sogar so vor, als ginge es wie in „Rote Korallen“ auch fu“r sie „nach Petersburg“ (EB, 28).

Die neuen Wohnorte weisen in beiden Fa"llen ein „verfremdete[s] Ambiente“ (Downes 2000, 636) auf. Die verunsicherte Effi befindet sich in einem ,verwunschene[n] Haus“ (EB, 79) und in „Rote Korallen“ erlebt die Urgroßmutter Sankt Petersburg als „ein[en] tiefe[n], da“mmerige[n] Traum“ (SS, 12). Betont werden zudem in beiden Texten Heimweh und Einsamkeit der Protagonistinnen (EB, 76, 89; SS, 12), sowie ein Gefu"hl der Ka"Ite, das weniger dem nordo"stlichen Klima als der emotionalen Distanz zum ku"hlen Ehemann geschuldet ist: So beklagt sich Effi bekanntlich, dass Innstetten „frostig wie ein Schneemann“ “(67) sei, und u“ber die Urgroßmutter in „Rote Korallen“ heißt es: „Sie wa“"rmte ihre fro“stelnde Seele an den glu“henden Herzen ihrer Liebhaber“ (SS, 14).

Auf diese Konstruktion einer parallelen Ausgangssituation zu Fontanes Roman folgt dann die deutliche Gestaltung zum Gegenentwurf, der besonders auf den Bereich der Geschlechterbeziehungen fokussiert ist. Anders als im Fall von Effi, für die der Ehebruch „eine unbewusste Auflehnung“ (Do 2003, 187) darstellt, wehrt sich die Urgroßmutter aktiv gegen die patriarchale Kontrolle ihres Ehemanns u“ber sie, indem sie wiederholt die Ehe bricht und ihren Gatten schließlich selbstbewusst mit ihrer als Widerstand gegen die sozialen Erwartungen kodierten Untreue konfrontiert. Wa"hrend Fontanes Protagonistin ,alles andere als eine selbsta“ndigmoderne Frau“ (Wende 2000, 154) ist, stellt die rebellische Urgroßmutter ,an emancipated Effi Briest figure“ (Littler 2007, 190) dar. Ihr Verhalten muss als ein Akt von fru"hfeministischem empowerment verstanden werden und la"sst sich als intertextueller Einspruch gegen Effi Briest und ihren Autor werten, der mit seinem Portra"t der an den preußischen Konventionen scheiternden ,arme[n] Effi“ (EB, 292) zwar die negativen Implikationen der streng regulierten bu"rgerlichen Ehe hervorhebt, aber nicht die patriarchale Geschlechterordnung an sich in Frage stellt.

„Fontane inszeniert mit dem Paar Effi-Innstetten das Muster einer preußischdeutschen Standesehe und verteilt dabei paradigmatisch die Rollen“ (Andermatt 2000, 191), indem er in Effi Briest zeittypische Repra"sentationen von Ma"nnlichkeit und Weiblichkeit entwirft, die dem Mann die Spha“re von Geist und Kultur, der Frau hingegen den Bereich der Natur und des Elementaren zuordnen, der als im Widerstreit mit der rationalen gesellschaftlichen Ordnung befindlich dargestellt wird. Effi wird vom Autor deutlich in dieses ma“nnlich bestimmte „Weiblichkeitsmuster“ (192) eingeordnet, die ihr zugeschriebene Eigenschaft als „Naturkind“ (EB, 37) wird wiederholt hervorgehoben. Fontane hinterfragt in Effi Briest die zeittypischen Geschlechterzuordnungen nicht, obwohl er ihren problematischen Bildcharakter erkennt (Stephan 1981, 140-142). Vielmehr beteiligt er sich ,insgeheim selbst an der Konstruktion von Weiblichkeit“" (Erhart and Hermann 2003, 504) und somit an der Verfestigung konventioneller Geschlechterkonzepte. Dieser Perpetuierung des patriarchalen Gender-Diskurses in Fontanes Roman wird bei Hermann eine weiblich bestimmte Gegenversion an die Seite gestellt.

Mit dieser dialektischen Verwendung von Intertextualita"t steht Hermann deutlich in der verbreiteten Tradition von kritischen rewritings, welche die im

${ }^{5}$ EB wird als Sigel für Effi Briest verwendet (Fontane 1974, Abt. I, Bd. 4). 
abendla"ndischen Literaturkanon marginalisierten Gruppen ins Zentrum eines revisionistischen Textes stellen und ihnen so eine Stimme verleihen. Das geschieht insbesondere im Rahmen postkolonialer und feministischer Literatur (Allen 2000, 4-5), und versta“ndlicherweise sind es besonders Texte des patriarchalen und kolonialen neunzehnten Jahrhunderts, die Autoren und Autorinnen des spa"ten zwanzigsten Jahrhunderts zur intertextuellen Auseinandersetzung reizten (Sanders 2005, 124). Besonders klar zeigt sich Hermanns Ablehnung des von Fontane pra“sentierten Frauenbildes und die Einordnung von „Rote Korallen“ in die Tradition eines feministisch motivierten writing back in der „radikale[n] Umschreibung “ (Stephan 2002, 548) eines für Fontanes Werk zentralen narrativen Bezugsrahmens bei der Darstellung der Geschlechterthematik: dem Wasserfrauen-Motiv.

Die Figur der Meerjungfrau, Nixe oder Sirene ist als ,eine zentrale literarische Imagination der abendla“ndisch-patriarchalen Kultur“" (Stuby 1985, 69) und als „Metapher fu“r das Naturhafte, der ma“nnlichen Akkulturation Widerstrebende“ “ (71) Teil des bu“rgerlichen Gender-Diskurses. In den Romanen Fontanes, der besonders von der Melusine-Sage ,,zeit seines Lebens fasziniert“ “ war (Steinka“mper 2007, 343), stellt die Meerjungfrau ein wiederkehrendes Motiv dar. Auch Effi, die in den fru"hen Entwu“rfen des Romans noch „Betty von Ottersund“ “ heißen und so die Assoziation mit dem Wasser schon im Namen tragen sollte (Fontane 1974, Abt I, Bd. IV, 713), reihte er in die große Gruppe seiner Melusinenfiguren ein.

Für Effi geht von der See eine Anziehungskraft aus, die ambivalent besetzt ist und ihre Position „im Spannungsfeld zwischen gesellschaftlichen Maximen und privatem Glu“cksanspruch“ (Wende 2000) symbolisiert. Wiederholt wird „Effis preka“re Beziehung zum tru“gerischen Element des Wassers“" (Downes 2000, 637) betont und eine Verbindung zwischen dem Regellosigkeit und natu“rliche Wildheit symbolisierenden Wasser und Effis spa"terem Verstoß gegen die Bestimmungen der bu“rgerlichen Ehe hergestellt. Effis Affa“re mit Crampas entfaltet sich folgerichtig unter dem starken Einfluss des Meeres, und bei der Verfu“hrungsszene im Schlitten meint Effi gar ,„die Meerjungfrauen singen [zu] ho“ren“ “ $(\mathrm{EB}, 157)$.

Im Gegensatz dazu begegnet dem Publikum bei Hermann eine Figur, die sich offenbar der gela"ufigen Weiblichkeitsbilder deutlich bewusst ist, sie sich subversiv aneignet und sich in ihrer feministischen Rebellion als selbstbewusste Sirene pra“sentiert. Schon der Titel „Rote Korallen“ verweist eindeutig auf das Wasserfrauen-Motiv und seine literarischen Gestaltungen, insbesondere auf den Schmuck von Fouque's Undine (Stephan 2002, 549). Die Vorbereitung auf die Konfrontationsszene mit ihrem Ehemann zeigt die Urgroßmutter deutlich in ihrer Selbst-Inszenierung als Loreley-Figur und femme fatale: „Meine Urgroßmutter [saß] vor dem Spiegel in ihrem Schlafzimmer und ka“mmte sich ihr helles Haar“ " (SS, 15). Die Wahl des Schauplatzes entspricht den durchga“ngigen maritimen Allusionen der Erza“hlung. Im auf ehemaligem Sumpfgebiet erbauten Sankt Petersburg zieht sich die junge Frau in eine vom Wasser beherrschte soziale Gegenwelt zuru“ck, in der sie lebt „wie auf dem Grunde des Meeres“ “ (12). Auf diese Weise stilisiert sie sich zu einer enigmatischen, außerhalb der Gesellschaft stehenden Nixenfigur, die sich gegen die frauenfeindlichen restriktiven Normen des neunzehnten Jahrhundert wehrt, wie sie bei Fontane eindringlich literarisch gestaltet sind. Hermann nimmt 
hier zugleich Fontanes Kritik an den Zwängen der bürgerlichen Ehe auf und wendet sich gegen seine distanzlose $U$ ' bernahme von Geschlechterbildern und Gender-Normen.

Dementsprechend nimmt bei Hermann die Urgroßmutter, ganz anders als die an den Folgen ihres Ehebruchs zerbrechende Effi, nach dem Tod ihres Ehemanns ihr Leben selbst in die Hand. Mit ihrem neugeborenen Kind, das vermutlich aus dem Ehebruch, nicht aus der Ehe hervorgegangen ist (18), besteigt die Urgroßmutter entschlossen die Eisenbahn zuru“ck nach Berlin, anstatt wie Effi den Zu"gen in die Brandenburgische Heimat mit ,einer herzlichen Sehnsucht“ (EB, 89) nachzusehen.

Die politische Dimension der hier geschilderten emanzipatorischen Handlungsweise wird hervorgehoben durch die Einbettung der Erza"hlung in den historischen Kontext der russischen Revolution von 1905, die zu dem Zeitpunkt ausbricht, als die Urgroßmutter in ein neues, selbstbestimmtes Leben aufbricht. Die Gesetze und Normen, die die Unfreiheit der Ehefrau bedingen, werden so zur Metonymie für die bürgerliche Gesellschaft des 19. Jahrhunderts, genauer gesagt fu“r den preußischdeutschen Staat mit seinen restriktiven Konventionen und Regeln.

Die Figur der Meerjungfrau ist zentral beteiligt an Hermanns intertextueller Verschra“nkung von Kritik am patriarchalen Weiblichkeitsdiskurs mit einer Verurteilung der repressiven politischen Verha“ltnisse, denn in Fontanes Romanen stellen die Melusinen, die sich in die „prosaische Enge der preußischen Gesellschaft“ " nicht einfu“gen ko“nnen (Ziegler 2000, 183), eine Bedrohung der bisher gu“ltigen Ordnung im Allgemeinen und des sozio-politischen Systems des Kaiserreichs im Besonderen dar. „Die Herausforderung Preußens durch die Wasserfrau im Salon“ (Briegleb 2000, 110) besteht in dem zersto“rerischverfu“hrerischen Chaospotenzial, das die dem Elementaren verhafteten Melusine-Gestalten der patriarchalen rationalen Sozialordnung entgegensetzen.

Diese rebellische Energie verko“rpert die Urgroßmutter in Hermanns Erza“hlung, und sie gibt sie traditionsbildend an die nachfolgenden Generationen weiter, wodurch ein narrativer Bogen historischer Kontinuita"t vom neunzehnten bis zum spa"ten zwanzigsten Jahrhundert gespannt wird. Sich in diesem Zusammenhang auf Fontane als repra“sentativen Autor des ausgehenden neunzehnten Jahrhunderts zu beziehen, liegt nahe, und Hermann ordnet sich auch hier wieder in eine bestehende intertextuelle Tradition ein. Sie wird bestimmt von Werken wie F. C. Delius’ Die Birnen von Ribbeck (1991), besonders aber von Gu“nter Grass’ kontroversem Roman Ein weites Feld (1995).

Beide Bücher kontrastieren über die Verbindung zu Fontane die politischen Verha“ltnisse der deutschen Einheit von 1990 mit der Reichsgründung von 1871. Wa“hrend sich jedoch Delius in seinem monologartigen Buch einer fast ethnographischen Anna“herungsweise bedient, welche die von kolonialistischen Tendenzen gepra“gten Vorga“nge des deutschen Einigungsprozesses aus authentisch anmutender ostdeutscher Perspektive beleuchtet (von Oppen 2001) und Grass in seinem Roman ein weitschweifendes zeitgeschichtliches Panorama der Wendejahre entwirft, wa“hlt Hermann bezeichnenderweise die knappe Form einer Kurzgeschichte, die so stark mit ma“rchenhaften Zügen durchsetzt ist, dass sich ihr politischer Gehalt nur schwer erschließt. In den zahlreichen phantastischen Elementen der Erza“hlung a“ußert sich einerseits Hermanns Skepsis gegenu“ber der Geschichtsschreibung und wird

\section{3}


andererseits sowohl die von historischem Unversta“ndnis gepra“gte Position der Protagonistin als auch die diffus bedrohliche Einwirkung der Vergangenheit auf ihr Leben betont. Die unwahrscheinlich langlebige Urgroßmutter, deren Anwesenheit für die Erza“hlerin zunehmend bedra“ngend wird, erscheint dementsprechend als hexenhafte Alte, die ,mit knochiger Hand“ (SS, 18) aktiv in das Leben der Hauptfigur eingreift.

Diese außerordentlich dominante Urgroßmutter hat die Geschichte ihrer Rebellion zu einem zentralen Inhalt des Familiengeda“chtnisses gemacht. Sie vererbt das Korallenarmband, das Symbol ihrer erfolgreichen Emanzipation, als ,,affective memory icon“ " den nachfolgenden Generationen und hat es zum Zeitpunkt der Haupterza“hlung an die Erza“hlerin weitergegeben. Die mit dem Schmuckstu“ck verbundene Geschichte der Urgroßmutter bildet für die Familie einen fundamentalen Teil ihres kollektiven Geda"chtnisses und erfu"llt als solches eine identita"tsbildende Funktion.

Diese Fo“rderung „kollektiver Identita“tsbildung“ durch Entwicklung einer Erinnerungskultur und durch die Tradierung bestimmter Geda“chtnisinhalte stellt fu“ $r$ Gruppen jeder Art einen zentralen Vorgang dar, wie die Geda"chtnisforschung hervorgehoben hat (Assmann 2007, 25). Hermanns Erza“hlung hinterfragt die Gu“ltigkeit solcher familia“ren Geda“chtnistraditionen im Kontext der deutschen Geschichte. Sie verweist auf die stets vorhandene Selektivita“t dessen, was weitergegeben wird, deutet den Zusammenhang von „Kanon und Zensur“ an, den die Erinnerungsforschung als zentralen Prozess des „kulturellen Geda“chtnisses“ identifiziert hat (Assmann und Assmann 1987) und betont die Probleme, die sich fu“r die nachfolgenden Generationen aus dieser ,strukturellen Amnesie“ ergeben.

Hermanns Protagonistin identifiziert sich zu Beginn der Erza“hlung so stark mit den von der Urgroßmutter tradierten Inhalten des Familiengeda“chtnisses, dass sie kaum unabha“ngig von ihnen existieren kann: „Die Vergangenheit war so dicht mit mir verwoben, daß sie mir manchmal wie mein eigenes Leben erschien. Die Geschichte meiner Urgroßmutter war meine Geschichte. Aber wo war meine Geschichte ohne meine Urgroßmutter?“ (SS, 22). Ganz a“hnlich empfindet der Protagonist in Ein weites Feld, der als Fontanes Wiederga“nger ,die Wirklichkeit mit seiner perso“nlichen Imitation dieser Wirklichkeit zu verwechseln beginnt“" (Balzer 2001, 11). In beiden Texten verweist die schwache Identita“t der in der Gegenwart befindlichen Figuren auf eine u“berma“chtige Vergangenheit, deren kontinuierliches Einwirken auf die Jetztzeit als problematisch begriffen wird.

Hermanns Erza“hlerin reagiert auf diesen Umstand mit dem zo“gerlichen Versuch, sich zuna“chst der vertrauten Version der Familiengeschichte, die zugleich Teil der deutschen Vergangenheit ist, zu vergewissern und dann die Existenz verschwiegener Erinnerungen zu erkunden. In der Absicht, den „Petersburger Geschichten“ (SS, 19) auf den Grund zu gehen, wendet sie sich einer Seitenlinie der Familiengeschichte zu, die signifikanterweise wiederum intertextuelle Verbindungen zu Effi Briest aufweist. Eng verbunden mit der Geschichte der Urgroßmutter und ihrer Nachfahren ist das Schicksal von Isaak Baruw, dem Sekundanten des

${ }^{6}$ So Littler (2007, 190), die diese Bezeichnung von Anne Fuchs übernimmt. Dieser Begriff stammt von John A. Barnes, vgl. Pethes $(2008,126)$.

\section{3}


geto"teten Ehemannes, der mit Hilfe der jungen Witwe in den russischen Revolutionswirren nach Berlin floh, wo er ihr zeit seines Lebens treu ergeben blieb, wie sie stolz berichtet. Wie die Beschreibung dieses treuen Gefolgsmannes als ,bucklig und krumm“ (SS, 17) erkennen la“sst, ist Baruw als intertextuelle Bezugsfigur zu Alonzo Gieshu“bler, Fontanes „,kleine[m], schiefschultrige[m]“ “ (EB, 62) Apotheker konzipiert, der Effi glu“hend verehrt und am liebsten fu“r sie „ka“mpfen und sterben“ (EB, 65) würde.

Die miteinander verflochtenen Geschichten der beiden Familien setzen sich in die Gegenwart fort, als die Erza“hlerin den letzten Abko“mmling der Baruws, Isaaks „einzige[n] Urenkel“ “ (SS, 19) zu ihrem Geliebten nimmt, in der vergeblichen Hoffnung, sich auf diese Weise der master narrative ihrer Vorfahren zu vergewissern. Aber dieser sichtlich depressive oder gar traumatisierte Mann, der als ein stummer Fisch beschrieben wird, weigert sich, mit ihr über die gemeinsame Familienvergangenheit zu sprechen, und zieht so die von der Urgroßmutter tradierte Fassung indirekt in Zweifel. Auch ein Besuch beim Psychiater ihres Geliebten bleibt ergebnislos für die Protagonistin, und so scheitert sie letztlich bei ihrem Versuch, die hinter Ma"rchenelementen verborgene Realita“t der Familiengeschichte zu enttarnen und den Familienmythos durch Fakten zu ersetzen.

Was der Erza"hlerin selbst verborgen bleibt, offenbart sich allerdings dem aufmerksamen Leser. Das in der Tradition eliminierte Element der Familiengeschichte ist die europa“ische Judenverfolgung (Stephan 2002, 553). Der durch seinen Vornamen als Jude gekennzeichnete Isaak Baruw war 1905 ho“chstwahrscheinlich nicht prima“r auf der Flucht vor den Revolutionswirren, sondern fu“rchtete in den damaligen Pogromen um sein Leben (Nobile 2010, 291). Seine Reise mit „,dem deutschen Zug“ “ (SS, 18), die ihn vor der Verfolgung in Russland rettet, la“sst sich zugleich als Vorausdeutung auf die knapp vierzig Jahre spa“ter stattfindenden Bahntransporte in die Vernichtungslager lesen und erkla“rt, warum in der Familie Baruw auf ma“rchenhaft fruchtbare „,sieben Kinder und [...] sieben Enkelkinder“"

(19) nur ein einziger Urenkel folgte. Der Holocaust und die Baruws, die ihm zum Opfer fielen, sind das verschwiegene Element in der ma“rchenhaft verfremdeten und gescho“nten Geschichte der Urgroßmutter, die von perso“nlicher Revolte und Solidarita“t, aber nicht von menschlichem Versagen angesichts von Verfolgung oder gar von der Verstrickung in Schuld berichtet.

Dass Hermann diese Geschichte unter fortlaufender Bezugnahme auf Effi Briest erza“hlt, dient dazu, die enge Verbindung der beiden Zweige der Petersburger Geschichten zu verdeutlichen und zudem die Problematik der Judenverfolgung nicht nur in der Zeit des Nationalsozialismus und den Pogromen des zaristischen Russland zu verorten, sondern bewusst auf die Kontinuita"t antisemitischer Traditionen seit dem spa"ten neunzehnten Jahrhundert zu verweisen-wie sie sich bei Fontane als typischem Repra“sentanten seiner Zeit auch finden lassen (Fleischer 2009). Angesichts des Umstandes, dass ,Judenfeindschaft [...] ein kultureller Code [war], u“ber den sich die bu“rgerliche Gesellschaft des Kaiserreiches versta“ndigte“ “, ist in der Fontane-Forschung sogar vom ,selbstversta“ndliche[n] Antisemitismus“ “

${ }^{8}$ Wie Aleida Assmann betont, bedeutet Trauma ,die Unmo“glichkeit der Narration“ (2006, 264). 'Auf weitere versteckte Holocaust-Referenzen im Text weist Nobile hin $(2010,294)$. 
123 
des Autors gesprochen worden (Balzer 2000, 199). Auch Grass geht in Ein weites Feld auf Fontanes ambivalente Haltung Juden gegenu"ber ein, die aus heutiger Perspektive in Verbindung mit der „Endlo“sung“ (Grass 1995, 61) gebracht werden kann.

In Fontanes Romanen zeigt sich die vom Autor weitgehend akzeptierte gesellschaftliche Randstellung der Juden bezeichnenderweise gerade auch in ihrer Assoziation mit den ebenfalls ausgegrenzten Melusine-Figuren. Besonders im Fru"hwerk gibt es mit Wasserfrau-Zu"gen ausgestattete Frauengestalten, bei denen ,,das spezifisch Melusinenhafte—also das ,noch-nicht-ganz-Mensch-Sein' [...] unverkennbar Teil des Ju"dischen " (Balzer 2000, 198) ist. Diese Verbindung zwischen zwei marginalisierten Gruppen, den Frauen und den Juden, u“bernimmt Hermann und betont sie auf strukturelle Weise mit weiteren intertextuellen Verweisen, die Baruws Urenkel zu Effi Briest in Beziehung setzen. Wie Fontanes Protagonistin bewohnt der Geliebte eine Wohnung, die „,auf den Friedhof hinaus“ “ geht (SS, 20; EB, 259)—was in beiden Fa”llen auf den baldigen Tod der Figur vorausdeutet. Besonders aber lassen sich die Melusinenhaften Zu"ge, die Effi aufweist, in dem Geliebten in modifizierter Form wiederfinden. Diese Figur fa“"llt am sta“rksten aus der Realita”t der Gegenwart heraus und ist daher am deutlichsten von den phantastischen Elementen der Erza“hlung gepra“gt. Er erscheint „,wie ein Fisch. Er hatte fischgraue Augen und eine fischgraue Haut, er war wie ein toter Fisch“ “ (18-19).

In dieser ma“"rchenhaft ungreifbaren Fisch-Gestalt scheinen die sozialen Ausgrenzungsmechanismen, auf die Hermann unter Bezugnahme auf Fontane verweist, eine extreme, spa"te Verko"rperung zu finden. Der junge Baruw kann sich von der transgenerationellen Traumatisierung, unter der er leidet, nicht befreien und auch die Erza"hlerin fu"hlt sich u"berfordert vom schwierigen Umgang mit der Familiengeschichte, die als Metonym für die deutsche Vergangenheit steht.

Am Ende zerreißt die Protagonistin das Korallenarmband und zersto“rt so symbolisch die mu"hsam von der Urgroßmutter errichtete Ordnung der selektiven Familiengeschichte. Sie empfindet aber das Aufgeben dieses Familienerbstu"cks genauso als Verlust wie den unmittelbar folgenden Tod des jungen Baruw. Ihre Handlungsweise stellt ein hilfloses Aufbegehren gegen eine Geschichte dar, die sich ihr bis zuletzt entzieht und von der sich deshalb auch nie wirklich befreien kann. Dies scheint zuna“chst im Gegensatz zu der eindrucksvollen Meeresmetaphorik zu stehen, die ihren Befreiungsversuch begleitet: „Das Wasser rauschte, brandete, sang und stieg und schwemmte die Geschichten mit sich fort, die Stille und die Korallen, schwemmte sie zuru"ck [...] an den Meeresgrund " (28). Letztlich versta"rkt der erneute Ru"ckgriff auf das fluide Element des Wassers aber wiederum die empfundene Konturenlosigkeit und Unsicherheit, mit der die Vergangenheit durchga"ngig assoziiert wird. Es ist der Protagonistin am Ende nicht gelungen, die alten Geschichten zu entlarven und durch eine Wahrheit zu ersetzen, die ein gemeinsames Leben mit dem jungen Baruw ermo"glicht. Die Kommunikation scheitert sowohl im Austausch der Generationen als auch zwischen den Nachkommen der Opfer und der Ta"ter (Stephan 2002, 554). Es gibt in Sommerhaus, spa“ter keine Aussicht auf „Heilung‘ im Sinne einer Verso“hnung mit der deutschen Vergangenheit und ihren bis heute nachwirkenden Machtstrukturen. 
Bezeichnenderweise betrifft diese Fortwirkung gesellschaftlicher Repressionen in die Gegenwart hinein sowohl das verschwiegene Thema des Antisemitismus als auch die patriarchalen Geschlechterbeziehungen. Obwohl die nixenhafte Geschichte der Urgroßmutter sich als matriarchaler Gru“ndungsmythos pra"sentiert und als solcher nicht nur intertextuelle Verbindungen zu Fouque' und Fontane, sondern auch zu Ingeborg Bachmanns feministischem Prosatext „Undine geht““ aufweist (Bauer 2009, 69), ist argumentiert worden, dass nicht erst die Sinnsuche der Protagonistin in einer fruchtlosen Revolte gipfelt, schon die idealisierte Auflehnung der Urgroßmutter kann man als eigentlich gescheitert betrachten. Das Korallenarmband, das als Memento der Befreiung der Urgroßmutter aus patriarchalen Zwa”ngen dient, ist zugleich ,,a well established symbol within the bourgeois, male-dominated order“", so dass sich behaupten la“sst, dass die Rebellion der jungen Ehefrau letztlich innerhalb des Unterdru“ckungssystems verbleibt, gegen das aufbegehrt wird (56).

So ergibt sich der Befund einer erfolglosen Befreiung von der Geschichte. Das naive Verlangen der Erza“hlerin aus ,,[den] alten Geschichten [...] hinaus und fortgehen zu ko“nnen“ “(SS, 24), weil sie ihr ,das Leben schwer [machen] “ (26), ist gescheitert. In dieser Betonung einer Geschichte, die sich nicht abschu“tteln, sich aber auch nicht aneignen oder bewa"ltigen la“sst, liegt ein zentraler Aspekt der intertextuellen Verweise auf Fontane. Die sta“ndige Ru“ckbindung an den Gründerzeitautor pra“sentiert ihn als Gewa“hrsmann einer kritisch begriffenen historischen Kontinuita"t und instrumentalisiert ihn in einer Art und Weise, wie sie sich vergleichbar auch bei Grass in Ein weites Feld beobachten la“sst.

Verbunden durch die Hauptfigur, den als Wiederga“nger Fontanes gestalteten Theo Wuttke, werden bei Grass die politischen Hintergru“nde der Reichsgründung und der Wiedervereinigung parallel gefu"hrt und wird in kritischer Weise das Fortbestehen repressiver deutscher Zusta"nde u“ber die Jahrhunderte hinweg aufgezeigt. In Ein weites Feld dient prima“r das mechanische Bild des Paternoster ,,als Dingsymbol fu“r das Auf und Nieder der Geschichte, fu"r sich abwechselnde Regime und Zeitla"ufte" (Balzer 2001, 212), die von der Gru“nderzeit bis zur Gegenwart keine positiven Vera“nderungen ermo“glichen. Zudem erscheint das Wasser als Element der Geschichte, das von den Figuren im Ruderboot aufgewu“hlt und metaphorisch durchpflu“gt wird, am Ende aber wieder unvera"ndert glatt daliegt (Preece 2004, 204). Auch in diesem Punkt gleicht Hermanns Erza“hlung also dem Roman von Grass, allerdings nimmt das Wasser bei ihr eine ungleich wichtigere, strukturgebende Position ein und entwickelt eine komplexere polyvalente Semantik, die auf den Band Sommerhaus, spa“ter als Ganzes ausgeweitet wird (Littler 2007, 189-190).

Sowohl bei Hermann als auch bei Grass ist die Existenz der Figuren, die geradezu in der Geschichte gefangen sind, von einer Identita"tskrise begleitet, die auf den oben skizzierten, von Aleida und Jan Assmann beschriebenen Geda“chtnisprozessen beruht. Hermanns Protagonistin ist an die unzuverla“ssige Geschichte der Urgroßmutter gefesselt, und der „Fonty“ genannte Wuttke fu“hlt sich ,,weitgehend in der Biographie Fontanes gefangen“" (Thesz 2003, 445). In beiden Werken steht am Ende ein Ausbruchsversuch aus zeitgeschichtlichen und zugleich biographischen Zusammenha“ngen, dessen Gelingen bei Grass zweifelhaft ist und auch bei Hermann in Frage gestellt wird. Wie bei Grass der Paternoster, so la“uft auch Hermanns 
Geschichte letztlich im Kreis und endet bezeichnenderweise mit einer Frage, die schon im ersten Absatz der Erza“hlung thematisiert wurde: „War das die Geschichte, die ich erza“hlen wollte“ (SS, 29).

Die ausgepra“gte Metatextualita"t der Erza“hlung, die mehrfach betont wird, verweist, gepaart mit den oben beschriebenen Elementen der unzuverla"ssigen Narration, erneut auf die Fragwu"rdigkeit des Dargestellten. Einerseits befindet sich Hermann mit dieser Einscha"tzung der Geschichte als zirkula"r und der Geschichtsschreibung als fiktional in der postmodernen Tradition des New Historicism (White 1978), der die Trennung von Dokumentation und Fiktion als undurchfu"hrbar ablehnt und daher die Existenz einer objektiven, historischen Wahrheit bestreitet. Andererseits bewegt sich Hermann, indem sie „Geschichte“ immer zugleich als Historie und als Narration begreift, aber auch in ansatzweise bereits von Fontane abgesteckten Bahnen. Gerade in Effi Briest nimmt das Wort „Geschichte“ vieldeutige Konnotationen an und belegt, wie sehr es sich bei diesem Roman um eine ,self-reflective narrative“ handelt (Chambers 1995, XVIII).

Diese von Unsicherheit gepra"gte Situation, in der sich Hermanns Figuren befinden, wird in ihrem Gesamtwerk am deutlichsten in „Rote Korallen“ " mit der historischen Dimension in Verbindung gebracht. Entsprechend der bereits erwa“hnten Funktion als programmatisches „Motto“, die diese Erza“hlung fu“r den Band Sommerhaus, spa“ter einnimmt, artikuliert die Anfangserza"hlung besonders klar eine Problematik, die fu"r das gesamte Buch zur impliziten Voraussetzung wird. Auf ha"ufig unausgesprochene Weise sind alle Figuren bei Hermann von den Existenzbedingungen einer wurzellosen Gegenwart betroffen, die sich aus einer historischen Verunsicherung speist.

Vergleichsweise klar wird diese Auseinandersetzung mit historischen Fragestellungen noch in zwei weiteren Erza"hlungen des Bandes thematisiert. Es handelt sich um Texte, die im Band a"hnlich strategisch platziert sind wie die Anfangserza"hlung: „Sommerhaus, spa“ter“ gibt der Textsammlung den Namen und „Diesseits der Oder“ bildet die abschließende Erza“hlung. Diese Anordnung betont erneut, dass historische Zusammenha"nge bei Hermann durchaus eine intensive Bescha"ftigung erfahren und in denjenigen Erza"hlungen des Bandes, welche die geschichtliche Dimension nicht offen thematisieren, als Hintergrund zu erga"nzen sind. Wie schon „Rote Korallen“ bescha"ftigen sich auch diese anderen beiden Erza“hlungen mit Fragen der deutschen nationalen Identita"t und mit dem historisch beeinflussten Lebensalltag der heutigen und fru“herer Generationen. „Diesseits der Oder“ schildert die desillusionierte Gegenwart ehemaliger 68er (Jager 2007, 41-43), und „Sommerhaus, spa“ter“" verleiht der Desorientierung von Hermanns Altersgenossen, als deren Sprachrohr man sie so ha”ufig bezeichnet hat, besonders deutlich Ausdruck.

In beiden Texten versuchen die Figuren in einem Nachwendedeutschland heimisch zu sein, dessen politische Entwurzelung und trostlose Unbestimmtheit sich nicht zuletzt in territorialen Kategorien a"ußern. Es handelt sich bei den Figuren jeweils um Westberliner, die in Brandenburg gestrandet sind—schon durch die Wahl der Region wird auch hier die Verbindung zu Fontane immer mitgedacht. Die in „Rote Korallen“ entfaltete Wassermetaphorik mit ihrer unterschwellig bedrohlichen Qualita"t wird hier fortgefu"hrt und dient dazu, einerseits die unsicheren politischen Verha"Itnisse nach der Wiedervereinigung zu charakterisieren und 
andererseits die perso"nlichen Krisen der Figuren widerzuspiegeln. Es ergibt sich so eine doppelte Deterritorialisierung in Hermanns Texten, die gleichermaßen die politische Dimension wie auch die wurzellose private Existenz der Figuren betrifft. ${ }^{10}$

In „,Diesseits der Oder“ , dessen Titel auf Fontanes Abschnitt „Jenseits der Oder“ in den Wanderungen durch die Mark Brandenburg anspielt, positioniert Hermann ihre Hauptfigur, den mit der Gegenwart unzufriedenen und unerwartet mit seiner Vergangenheit konfrontierten Autor Koberling, an einem geschichtstra“chtigen Ort. Im Oderbruch, einer Gegend, die heute an der lange umstrittenen Grenze zu Polen liegt, unter Friedrich II. erstmals trockengelegt wurde und als solchermaßen unzuverla“ssiges Terrain die Verunsicherung der ihrer Vergangenheit entfremdeten und in der Nachwende-Gesellschaft nur halb heimischen Protagonisten widerspiegelt, bewegt er sich durch eine alptraumartige Sumpflandschaft, deren mangelnde Substanz sich in wa“ssriger Form pra”sentiert.

In der Gegenwart, in der Hermanns Erza“hlung spielt, stellt das Oderbruch ein Territorium dar, das sich zu Zeiten der deutschen Teilung den Westberlinern entzog und nach der Wiedervereinigung vom Kontext einer westdeutschen ,Kolonisierung ‘ der ehemaligen DDR gepra“gt ist. Zu Fontanes Zeiten, als sich Preußen auch ,,jenseits der Oder“ erstreckte, bildeten die dort gelegenen Gebiete einen ,deutsch-slawischen Kulturgrenzraum“" (Ossowski 2000), der von einer aggressiven deutschen Expansionspolitik in Richtung Osten gepra“gt war, die spa“ter im Nationalsozialismus eine verbrecherische Zuspitzung erfahren würde. In Effi Briest wird das Thema deutlich angesprochen, wenn Innstetten sich aus kolonialistischer Perspektive kritisch u“ber die slawische Bevo“lkerung Hinterpommerns äußert und mit einem beunruhigten Fazit schließt, das genauso von Hermanns Figuren gea“ußert werden ko“nnte: „Hier ist alles unsicher" " (EB, 44-45).

Diese Unsicherheit zeigt sich in ,,Sommerhaus, spa“ter“ ' besonders deutlich in der fu“r Hermanns Figuren so typischen Ziel-und Orientierungslosigkeit und signalisiert eine bisher wenig beachtete Einbettung vom Lebensgefu“hl dieser Charaktere in eine Auseinandersetzung mit der deutsch-deutschen Vergangenheit und dem daraus erwachsenden Gegenwartsbefund. Daru“ber hinaus nimmt der Text auch deutlich die in „Rote Korallen“ entwickelte gender-Thematik wieder auf, die einen wichtigen Aspekt von Hermanns Auseinandersetzung mit Fontane darstellt.

Typischerweise pflegen Hermanns Figuren einen bohemienartigen Berliner Lebensstil, der einerseits von einer versuchten U berwindung der Geschlechtergrenzen und andererseits von Unverbindlichkeit und Bindungsscheu gepra“gt ist. Zugleich erwa"gen sie aber immer wieder andere, konventionellere und gebundenere Lebensformen und spielen „Sich-so-ein-Leben-vorstellen“ (SS, 31). Sie geben sich der meist unausgesprochenen $U$ berlegung hin, ihren promisken Alltag zugunsten von klassisch-bu“'rgerlichen Lebensentwürfen aufzugeben. Diese erscheinen jedoch nur sehr begrenzt als lebbare Optionen, denn sie sind bis heute mit den patriarchalen Strukturen weiblicher Unfreiheit und ma“nnlicher Kontrolle verbunden, gegen welche die Urgroßmutter in „,Rote Korallen“ a aufbegehrt hatte. Obwohl die Figuren

${ }^{10}$ Vgl. Littler (2007, 179, 189). Mit dem Begriff der Deterritorialisierung bezieht Littler sich auf Deleuze.

\section{3}


sich spu“rbar zu den Sicherheit versprechenden herko"mmlichen Strukturen hingezogen fu“hlen, siegt letztlich doch immer das Misstrauen diesen traditionellen Lebensformen gegenu“ber, wie die Protagonistin in „Sommerhaus, spa“ter“ “ eindrucksvoll demonstriert. Sie la“sst ihren Gelegenheitsgeliebten Stein, der fu“r sie ein altes Gutshaus renoviert, so lange auf eine Entscheidung für oder gegen eine konventionelle Zweierbeziehung mit traditioneller Geschlechterrollenverteilung warten, bis er schließlich frustriert das Haus niederbrennt.

Dass dieser halbherzig erwogene Neuanfang scheitert, der auch von Stein lediglich mit den zo“gerlichen Worten „,das hier ist eine Mo“glichkeit, eine von vielen“ (152) beworben wird, ist erneut in den tradierten Strukturen gesellschaftlicher Unfreiheit begru"ndet, die auch hier wieder ihren Einfluss geltend machen - und die wiederum in Beziehung zu Fontane gesetzt werden. Das la“sst sich klar schon an der Beschreibung des in einem trostlosen ma“rkischen Provinzdorf gelegenen Hauses ablesen. Man hat die feindseligen Mieter aus DDR-Zeiten vor kurzem aus dem Gebäude vertrieben und die aus Berlin angereiste Protagonistin fu“hlt sich unwohl auf diesem feindlichen, ,kolonisierten“ Gela“nde. Das Haus selbst ist eine wenig einladende Ruine, der man die urspru“ngliche Pracht eines eindrucksvollen preußischen Gutshauses mit Giebeldach und Veranda kaum noch anmerkt. Hier wird erneut die Verbindung zu Fontane herstellt, denn so a“hnlich muss man sich das ,schon seit Kurfu“rst Georg Wilhelm von der Familie von Briest bewohnte Herrenhaus zu Hohen-Cremmen“ vorstellen (EB, 7). Das verfallene Haus bietet auf diese Weise eine ersehnte aber zweifelhafte Zuflucht, weil es neben domestischer Idylle auch die repressive preußische Vergangenheit repra“sentiert. Hermann nimmt auch hier wieder die den Band durchdringende Wassermetaphorik auf und beschreibt das Haus als „ein in lange vergangener Zeit gestrandetes, stolzes Schiff“، (SS, 148). So wird erneut deutlich signalisiert, dass den Figuren ein fester, zuverla“ssiger Grund und Boden und eine ra"umlich verankerte Heimat verwehrt bleibt.

Indem Hermann das Berlin der Gegenwart und sein nun wieder zugängliches Umland mit den Bildern einer sich dem festen Zugriff entziehenden Wasserwelt beschreibt, pra“sentiert sie das vereinte Deutschland aus der Inselperspektive einer jungen Generation ehemaliger Westberliner, die in einer sich wandelnden Welt nach Heimat suchen und sich stattdessen konfrontiert sehen mit einem Herkunftsland, das so stark von seiner unverarbeiteten Vergangenheit gepra“gt ist, dass die Entwicklung einer stabilen nationalen und individuellen Identita“t auf kurze Sicht unmo“glich und auf lange Sicht fraglich erscheint. Ebenso ungewiss sind die Geschlechterbeziehungen, die weder in der für Hermanns Protagonisten typischen promisken Unverbindlichkeit noch in den von den Figuren teilweise abgelehnten, letztlich aber nicht eindeutig zurückgewiesenen Strukturen der klassischen heterosexuellen Zweierbeziehung positive Perspektiven bieten. $\mathrm{Zu}$ einer Verhandlung neuer Formen des Zusammenlebens kommt es in den Texten bezeichnenderweise nicht. Stattdessen unterwerfen sich die vom historischen Erbe gela“hmt erscheinenden Figuren einem Prozess des fortwa“hrenden Aufschubs, der in der semantischen Struktur des Buchtitels eloquent ausgedru“ckt ist.

Die Wassermetaphorik bleibt durchgehend eingebunden in dieses Lebensgefu“hl einer sinnentleerten Gegenwart ohne Zukunftsperspektive. Die „Beziehungslosigkeit, 
Orientierungssuche und melancholische Sehnsucht“" (Vollmer 2006, 59), die fu“r Hermanns Figuren typisch sind, werden am Ende von „Rote Korallen“ deutlich zusammengefu“hrt mit der pervasiven maritimen Bildlichkeit, wenn die Erza“hlerin sich fragt: „Ist es das? daß da na“mlich gar nichts ist? nur die Mu“digkeit und die leeren, stillen Tage, ein Leben wie das der Fische unter Wasser?، ‘(SS, 26).

Man hat die Beschreibung dieser „,mu“den, erscho“pften, ihrer Identita“t niemals gewissen Figuren“" (Blamberger 2006, 194) auf ein allgemeines Lebensgefu“hl der Postmoderne, auf die „,kulturelle Krise“ (186) der Jahrtausendwende zuru“ckgefu“hrt, die im fragmentierten Berlin der Nachwendezeit eine akute Auspra“gung erfa“hrt. Wie die bisherige Analyse gezeigt hat, sind das zweifellos Pha“nomene, von denen Hermanns Schreiben beeinflusst ist und auf die sich die vagen Aussagen und das konsequenzlose Verhalten ihrer Figuren zu einem gewissen Grad zuru“ckfu“hren lassen. Es ist zugleich behauptet worden, dass die „Ratlosigkeit“ und „Unentschiedenheit““ (186), von der Hermanns Werke gepra“gt sind, auf einem Mangel an Rebellion gegen die literarische Vorga“ngergeneration beruhten, was sich nicht zuletzt auch an Hermanns Umgang mit Intertextualita“t ablesen lasse. Anstatt antagonistisch gegen den Kanon anzuschreiben, gebe sie sich nur „,der Kunst des Sammelns“ " hin (189) und verwende als literarische Techniken „Re-Make und Re-Mix“ “ (192). Wenn man jedoch paradigmatisch betrachtet, in welcher Art und Weise sich Hermann in „Rote Korallen“ mit Effi Briest auseinandersetzt, wird deutlich, dass sich in ihren Erza“hlungen durchaus Ansa“tze von Gegenentwu“rfen beobachten lassen, die allerdings letztlich als undurchfu“hrbar entlarvt werden beziehungsweise sich als gescho“nte Fantasien entpuppen.

Nicht zufa“llig folgt auf die ma“rchenhaft-phantastische erste Erza“hlung in Sommerhaus, spa“ter nur noch im weitesten Sinne realistisches Erza“hlen. „Rote Korallen“ " legt die Parameter fest, auf denen die u“brigen Texte beruhen, ohne dass sie sich im Folgenden immer wieder klar manifestieren. In ihrer Eigenschaft als programmatisches Motto weist die Einleitungserza"hlung aus der Perspektive der Wendegeneration auf eine deutsche Tradition gesellschaftlicher Ausgrenzung und politischer Verfolgung hin, die bis ins neunzehnte Jahrhundert zuru“ckverfolgt wird. Hermann schildert die Existenzbedingungen ihrer Zeitgenossen in der Berliner Republik als wesentlich von den Geschehnissen der Nazi-Zeit beeinflusst, die ihr wiederum in mehrfacher Hinsicht als Fortsetzung preußischer Geschichte gilt. Die Unzula“nglichkeit der Gegenwart wird als Folge unu"berwundener und unu"berwindlicher historischer Strukturen begriffen. Der erfolglose Befreiungsversuch der Protagonistin in „Rote Korallen“" steht repra“sentativ fu“r ein desillusioniertes Unvermo“gen, sich mit Neuentwu“rfen gegen das unerledigte Erbe durchzusetzen.

Vor diesem Hintergrund muss man Hermanns Beziehung zu Fontane betrachten. Zuna“chst gilt er ihr als Repra“sentant sozio-historischer Gegebenheiten, deren Kontinuita“t sie als problematisch begreift und von denen sie die Gegenwart stark beeinflusst sieht. Daru“ber hinaus sind Fontanes Schreiben und seine Haltung den gesellschaftlichen Zusta“nden gegenu“ber von einer Zwiespa“ltigkeit gepra“gt, die Hermanns eigener Position nicht una"hnlich ist. Es handelt sich bei Fontane ja keineswegs um einen Autor, der die sexistischen und antisemitischen Tendenzen, gegen die Hermann sich wehrt, u“berzeugt vertritt. Zugleich ist er aber auch trotz seiner Verurteilung des ,tyrannisierende[n] Gesellschafts-Etwas“ “ (EB, 236) kein 
ganz eindeutiger sozialer Kritiker, sondern stellt sich heutigen Lesern ambivalent dar. Das zeigt sich charakteristischerweise auch bei Grass, der mit dem Titel seines Romans Ein weites Feld den beru"hmt vagen Ausspruch des alten Briest aufgreift. Es gibt keine klaren Schuldzuschreibungen, und, wie betont worden ist, bleibt eine Enttäuschung beim Lesen von Fontanes Werken mitunter nicht aus, denn er ,,ruft nicht unmittelbar zu Vera“nderung auf und bietet keine Lo“sungen an“ (Mu“ller-Seidel 1980, 480). Dieses Urteil erinnert an die a“hnlich pra“skriptive Kritik, die an Hermanns Erza“hlungen geu“bt worden ist und weist auf eine Vergleichbarkeit beider Autoren hin, die es sich na“her zu betrachten lohnt.

Martin Swales hat unter Bezugnahme auf Auerbach und Luka'cs von Fontanes „Halbheit“ gesprochen, die er besonders in Fontanes literarischer Konfliktvermeidung manifestiert sieht, die der Erza“hlerinstanz eine „taktvoll[e] und zuru“ckhaltend[e]“ Rolle (Swales 1989, 77) zuteilt und insgesamt die gesellschaftliche Ordnung sowohl kritisiert als auch verteidigt. Obwohl Fontane in den repressiven Regeln der preußischen Gesellschaft ,eine konstante Verletzung menschlicher Werte“ sah, ,glaubt[e] er, daß die Menschen ohne diese Ordnung nicht auskommen ko“nnen“ (78). Die Wirkung einer solch unentschiedenen Haltung zwischen Auflehnung und Anpassung ist eine spu“rbare Inauthentizita"t des Geschilderten, die sich auch im Empfinden der Figuren niederschla“gt. Besonders bei Effi la“sst sich das beobachten, die sich leidenschaftslos einer „bloßen Liebelei““ (Mu“ller-Seidel 1980, 372) hingibt und bea“ngstigend reale Konsequenzen fu“r etwas erfa“hrt, das ihr ,gleichsam vorfabriziert“ (Swales 1989, 78) erscheint. In der Tat gleicht Effi mit ihrem Handeln, das vage zwischen unausgesprochenen eigenen Wünschen und gesellschaftlichen Erwartungen positioniert ist, den Figuren in Hermanns Erza“hlungen. Sie sagt selbst, dass sie angesichts der Affa“re mit Crampas weder ,,rechte Reue“ noch „,rechte Scham“ empfinde (EB, 219), und zieht kurz vor ihrem Tod das bedauernde Fazit, dass sie vieles in ihrem Leben nur „beinah“ (EB, 280) gemacht habe.

Fontanes Intention, die leere Formelhaftigkeit der gesellschaftlichen Normen, welche die Ehe der Instettens sinnlos zersto“ren, zu entlarven, bleibt so auf halbem Wege stecken, weil eine authentische Lebenserfahrung der Figuren gleichzeitig auf der Strecke bleibt. Effi und Innstetten sind in dieser Hinsicht Vorla“ufer von Stein und seiner Freundin, die ebenso wie Fontanes Protagonistin auf das wahre Erlebnis warten, das nicht kommt. Auch Hermanns Figuren ringen um Authentizita“t, ko“nnen sich von den sozio-historischen Strukturen nicht frei machen und verharren deshalb in einem inaktiven Zustand sinnloser Erwa“gung von Optionen, die eigentlich schon verspielt sind. Fontane kann deshalb trotz seiner Zugeho“rigkeit zur Gründerzeit und seiner bestenfalls ambivalenten Haltung gegenu“ber sozialen Repressions-und Ausgrenzungsmechanismen, die sich als Vorboten des Faschismus begreifen lassen, für Hermann nicht nur ein antagonistischer Vorläufer sein, den es in intertextuellen Gegenentwu"rfen zu beka“mpfen gilt. Er offenbart sich zugleich auch als ein Autor, dessen Befindlichkeit Hermanns eigener Haltung stark verwandt ist.

Zusammenfassend und auswertend la“sst sich sagen, dass Hermann in Sommerhaus, spa"ter ein Bild vom Stand der deutschen Nation entwirft, das entscheidend von einer bedru“ckenden Vergangenheit beeinflusst ist, aus der es bis heute keinen Ausgang gibt, und die sich in die umstrittenen und von Teilung gepra"gten deutschen 
Territorien eingeschrieben hat. Die Gegenwart erscheint dementsprechend als unsichere Wasserwelt, in der fluide Identita"ten erfolglos navigiert werden und historische Dimensionen in erstaunlicher Weise unzuga“nglich bleiben. Der überraschend unwissende Umgang der Hauptfigur mit den niemals an die Oberfla“che dringenden Holocaust-Hinweisen in „Rote Korallen“ scheint dabei anzudeuten, dass Hermanns Generation einerseits der Konfrontation mit der Vergangenheit nicht aus dem Weg gehen kann. Anderseits stoßen die Figuren aber auf ein der o“ffentlichen Debatte widerstrebendes privates Schweigen und tun sich letztlich mit ihrer wahrscheinlichen Abstammung von der Gruppe der Ta“ter und Mitla“ufer schwer. Gewissheit u“ber die Vergangenheit gibt es in diesen Erza“hlungen nie, auch in „Diesseits der Oder“ dominieren das Vergessen und der Selbstbetrug, mit dem der Protagonist ein angedeutetes homosexuelles Jugenderlebnis verdra“ngen will (Nobile 2010, 309-310).

Um diese Fortwirkung althergebrachter und zugleich unaufgelo"ster und unzuverla“ssiger Strukturen zu betonen, verwendet Hermann einerseits die Unsicherheit betonende Wassermetaphorik, die alles im Fluss auflo"st, und stellt andererseits Verbindungen zu Theodor Fontane her. Der kanonische Autor wird gleichzeitig als Repra“sentant der problematischen deutschen Tradition des letzten Jahrhunderts eingesetzt, von der es sich eigentlich abzugrenzen gilt, und als literarischer Vorla"ufer begriffen, dessen Figuren auf a“hnliche Weise mit dem Pha"nomen einer aus sozialen Strukturen erwachsenen Inauthentizita“tzuka“mpfen haben wie Hermanns ziellose Protagonisten.

Letztlich offenbaren sich die oft kritisierte Haltung von Hermanns ziellosen Figuren und die „Poetik der Unentschiedenheit“ (Blamberger 2006), der sie entspringen, durchaus als Ausdruck einer neuen Generation, aber einer Generation, die stark von der Vergangenheit gepra"gt ist. Die Ereignisse der Wiedervereinigung spielen dabei eine zentrale Rolle, denn sie markieren nicht nur das Scheitern der gesellschaftlichen Utopie des Sozialismus, sie bedeuten auch eine (westdeutsche) Ru“ckkehr zu Orten und Strukturen, die zur Zeit der Teilung als aufgeschobene Konflikte und unerledigtes Erbe wenig Beachtung erfuhren und sich jetzt besonders ehemaligen Westberlinern wieder sta“rker ins Bewusstsein dra“ngen. Die wa“ssrige Vision von Brandenburg, die Hermann wiederholt gestaltet, ist ein eloquenter bildhafter Ausdruck dieser Problematik.

Hermanns Versuch, sich der Vergangenheit zu stellen, die sich ihrer Generation im Berliner Umfeld jetzt versta"rkt pra“sentiert, fu“hrt sie zu einem geradezu untrennbar mit der Region verbundenen literarischen Vorla“ufer, der zugleich als Repra“sentant seiner Epoche und ihres andauernden Einflusses fungiert. Ihre Auseinandersetzung mit Fontane, die unter rebellischen Vorzeichen beginnt, wie die Anti-Effi-Briest-Geschichte der Urgroßmutter in „Rote Korallen“ illustriert, weicht jedoch letztlich einem desillusionierten Einversta"ndnis mit Fontanes Einsichten in die gesellschaftlichen Mechanismen seiner Zeit. Hermann sieht sich genauso wenig wie Fontane im Stande, zu den bestehenden Zusta“nden einen Gegenentwurf zu formulieren und diesen mit U berzeugung zu vertreten.

Hermanns melancholische Zwischenzusta“nde und Fontanes halbherzige Gesellschaftskritik offenbaren sich letztlich gleichermaßen als ein aus Hilflosigkeit entsprungener Distanzmechanismus, der sich vielleicht am Treffendsten mit dem

\section{3}


Begriff der Ironie umschreiben la“sst. Wa“hrend sich bei Fontane diese Haltung auf klassische Weise auch in seinem ironischen Schreibstil a"ußert, la"sst sich in Hermanns Werken allerdings bis auf ganz wenige Ausnahmen (SS, 143) keine sprachliche Ironie verzeichnen. Stattdessen setzt sie ihren beru“hmten ,,elegisch[en] Grundton“ " (Schulze 1998), ein, der in angemessener Weise ihre Trauer u“ber die nicht wahrnehmbaren Chancen ihrer Figuren zum Ausdruck bringt.

\section{References}

Allen, G. (2000). Intertextuality. London: Routledge.

Andermatt, M. (2000). „Es rauscht und rauscht immer, aber es ist kein richtiges Leben.“ Zur Topographie des Fremden in Fontanes Effi Briest. In H. Delf von Wolzogen (Hg.), Theodor Fontane. Am Ende des Jahrhunderts (Bd. III, S. 190-199). Wu“rzburg: Ko“nigshausen \& Neumann.

Assmann, A. (2006). Erinnerungsra"ume. Formen und Wandlungen des kulturellen Geda"chtnisses. München: C.H. Beck.

Assmann, J. (2007). Das kulturelle Geda“chtnis. Schrift, Erinnerung und politische Identita“t in fru“hen Hochkulturen. München: C.H. Beck.

Assmann, A., \& Assmann, J. (Hg.). (1987). Kanon und Zensur. Beitra“ge zur Archa“ologie der literarischen Kommunikation II.Mu“nchen: Wilhelm Fink.

Balzer, B. (2000). „Zugegeben, daß es besser wa“re, sie fehlten, oder wa“ren anders, wie sie sind“ —Der selbstversta“ndliche Antisemitismus Fontanes. In H. Delf von Wolzogen (Hg.), Theodor Fontane. Am Ende des Jahrhunderts (Bd. I, S. 198-209). Würzburg: Ko“nigshausen \& Neumann.

Balzer, B. (2001). Geschichte als Wendemechanismus: Ein weites Feld von Gu“nter Grass. Monatshefte, 93(2), 209-220.

Bauer, E. K. (2009). Narratives of femininity in Judith Hermann's Summerhouse, later. Women in German Yearbook, 25, 50-75.

Biendarra, A. S. (2004). Gen(d)eration next: Prose by Julia Franck and Judith Hermann. Studies in Twentieth and Twenty-First Century Literature, 28, 211-239.

Blamberger, G. (2006). Poetik der Unentschiedenheit. Zum Beispiel Judith Hermanns Prosa. Gegenwartsliteratur, 5, 186-206.

Briegleb, K. (2000). Fontanes Elementargeist: Die Preußin Melusine. Eine Vorstudie zum Stechlin. In H. Delf von Wolzogen (Hg.), Theodor Fontane. Am Ende des Jahrhunderts (Bd. II, S. 110-121). Wu“rzburg: Ko“nigshausen \& Neumann.

Broich, U. (1985). Formen der Markierung von Intertextualität. In U. Broich \& M. Pfister (Hg.), Intertextualita“t. Formen, Funktionen, anglistische Fallstudien (S. 31-47). Tübingen: Max Niemeyer.

Burtscher, S. (2002). „Glu“ck ist immer der Moment davor“-_Judith Hermann: Sommerhaus, spa“ter. Gegenwartsliteratur der 90er-Jahre im Deutschunterricht. Der Deutschunterricht, 54(5), 80-85.

Chambers, H. (1995). Introduction. In T. Fontane, Effi Briest (S. VII-XIX). London: Penguin.

Delius, F. C. (1991). Die Birnen von Ribbeck. Reinbek bei Hamburg: Rowohlt.

Do, K.-S. (2003). Ehe und Ehebruch in der Literatur des 19. Jahrhunderts: Untersuchungen zu Gutzkow, Stifter, Büchner und Fontane. Berlin: Mensch-und-Buch-Verlag.

Downes, D. (2000). Effi Briest. In C. Grawe \& H. Nürnberger (Eds.), Fontane-Handbuch (pp. 633-651). Stuttgart: Kro"ner.

Erhart, W. \& Hermann, B. (2003). Feministische Zuga“nge_,,Gender Studies“،. In H. L. Arnold., \& H. Detering (Hg.), Grundzu“"ge der Literaturwissenschaft (S. 498-515). Mu“nchen: dtv.

Fleischer, M. (2009). Fontane und die Judenfrage. Berlin: Verlag fu“r Berlin Brandenburg.

Fontane, T. (1974). Werke, Schriften und Briefe. W. Keitel \& H. Nu“rnberger (Hg.). Mu“nchen: Carl Hanser.

Geiger, T. (2004). Interview mit Judith Hermann. Dimension ² 7, 46-59.

Grass, G. (1995). Ein weites Feld.Go“ttingen: Steidl.

Hermann, J. (1998). Sommerhaus, spa“ter. Frankfurt am Main: S. Fischer. Illies, F. (1998). Die Traumwandlerin. Frankfurter Allgemeine Zeitung, 17.10. 
Jager, B. (2007). Verwisch die Spuren! Judith Hermanns Sommerhaus, spa“ter als Lesebuch fu“ $r$ Sta“dtebewohner. Text \& Kontext, 29, 29-47.

Krekeler, E. (2003). Stille Wasser. Nicht tief. Ru“ckkehr einer Ikone. Judith Hermann erza“hlt neue Geschichten einer auslaufenden Generation. Die Welt, 31.01

Littler, M. (2007). Cultural memory and identity formation in the Berlin Republic. In S. Taberner (Hg.), Contemporary German fiction. Writing in the Berlin Republic (S. 177-195). Cambridge: Cambridge University Press.

Lo“ffler, S. (2009). Alice ha“lt auch schon mal ein Schla“fchen. Der Falter, 21.

Mu“ller-Seidel, W. (1980). Theodor Fontane. Soziale Romankunst in Deutschland. Stuttgart: Metzler.

Nobile, N. (2010). A ring of keys: thresholds to the past in Judith Hermann's Sommerhaus, spa“ter. Gegenwartsliteratur, 9, 288-315.

Ossowski, M. (2000). Fragwürdige Identita“t? Zur national-territorialen Bestimmung der Figuren aus dem deutsch-slawischen Kulturgrenzraum in Fontanes Spa"twerk. In H. Delf von Wolzogen (Hg.), Theodor Fontane. Am Ende des Jahrhunderts (Bd. I, S. 255-268). Würzburg: Ko“nigshausen \& Neumann.

Pethes, N. (2008). Kulturwissenschaftliche Geda“chtnistheorien. Hamburg: Junius.

Preece, J. (2004). The life and work of Günter Grass. Literature, history, politics. Basingstoke: Palgrave Macmillan.

Quartett, Literarisches. (2006). Gesamtausgabe aller 77 Sendungen von 1988 bis 2001. Berlin: Directmedia Publishing.

Sanders, J. (2005). Adaptation and appropriation. London: Routledge.

Schulze, I. (1998). Wo scho“ne Menschen lange warten. Die Welt, 24.12.

Shafi, M. (2009). Housebound: Selfhood and domestic space in narratives by Judith Hermann and Susanne Fischer. Amsterdamer Beitra“ge zur neueren Germanistik, 67, 341-358.

Steinka“mper, C. (2007). Melusine-vom Schlangenweib zur „Beaute’ mit dem Fischschwanz‘. Geschichte einer literarischen Aneignung. Go“ttingen: Vandenhoeck \& Ruprecht.

Stephan, I. (1981). „Das Natu“rliche hat es mir seit langem angetan.“ Zum Verha“ltnis von Frau und Natur in Fontanes Cécile. In R. Grimm \& J. Hermand (Hg.), Natur und Natu“rlichkeit. Stationen des Gru“nen in der deutschen Literatur (S. 118-149). Ko“nigstein/Ts: Athena“um.

Stephan, I. (2002). Undine an der Newa und am Suzhou River. Wasserfrauen-Phantasien im interkulturellen und intermedialen Vergleich. Zeitschrift für Germanistik, NF, 12, 547-563.

Stuby, A. M. (1985). Sirenen und ihre Gesänge: Variationen u“ber das Motiv des Textraubs. In A.M. Stuby (Hg.), Frauen: Erfahrungen, Mythen, Projekte (S. 69-87). Berlin: Argument-Verlag.

Swales, M. (1989). Mo“glichkeiten und Grenzen des Fontaneschen Realismus. In H. L. Arnold (Hg.), Fontane (pp. 75-87). Mu“nchen: Edition Text ? Kritik.

Taberner, S. (2005). German literature of the 1990s and beyond. Normalization and the Berlin Republic. Rochester, NY: Camden House.

Thesz, N. (2003). Identita“t und Erinnerung im Umbruch: Ein weites Feld von Gu“nter Grass. Neophilologus, $87,435-450$.

Vollmer, H. (2006). Die sprachlose Na“he und das ferne Glu“ck. Sehnsuchtsbilder und erza“hlerische Leerstellen in der Prosa von Judith Hermann und Peter Stamm. Literatur fu“r Leser, 29, 59-79.

von Oppen, K. (2001). „Wer jetzt schwarzweiß malt, hat keine Ahnung“": Friedrich Christian Delius‘s Die Birnen von Ribbeck and the predicament of Wendeliteratur. German Life and Letters, 54, 352-365.

Wende, W. (2000). „Es gibt ... viele Leben, die keine sind...“ Effi Briest und Baron von Innstetten im Spannungsfeld zwischen gesellschaftlichen Verhaltensmaximen und privatem Glu“cksanspruch. In H. Delf von Wolzogen (Hg.), Theodor Fontane. Am Ende des Jahrhunderts (Bd. II, S. 148-160) Würzburg: Ko“nigshausen \& Neumann.

White, H. (1978). The tropics of discourse. Baltimore: John Hopkins University Press.

Ziegler, E. (2000). Die Zukunft der Melusinen. Weiblichkeitskonstruktionen in Fontanes Spa“twerk. In H. Delf von Wolzogen (Hg.), Theodor Fontane. Am Ende des Jahrhunderts (Bd. II, S. 174-185). Wu“rzburg: Königshausen \& Neumann. 